\title{
Periodic Nonlinear Schrödinger Equation and Invariant Measures
}

\section{J. Bourgain}

I.H.E.S., 35, route de Chartres, F-911440 Bures-sur-Yvette, France

Received: 27 October 1993 / in revised form: 21 June 1994

\begin{abstract}
In this paper we continue some investigations on the periodic NLSE $i u_{t}+u_{x x}+u|u|^{p-2}=0(p \leqq 6)$ started in [LRS]. We prove that the equation is globally wellposed for a set of data $\phi$ of full normalized Gibbs measure $e^{-\beta H(\phi)} H d \phi(x), H(\phi)=\frac{1}{2} \int\left|\phi^{\prime}\right|^{2}-\frac{1}{p} \int|\phi|^{p}$ (after suitable $L^{2}$-truncation). The set and the measure are invariant under the flow. The proof of a similar result for the $\mathrm{KdV}$ and modified $\mathrm{KdV}$ equations is outlined. The main ingredients used are some estimates from [B1] on periodic NLS and KdV type equations.
\end{abstract}

\section{Introduction}

Consider the nonlinear Schrödinger equation (NLSE) in the space periodic setting

$$
i u_{t}+u_{x x}+u|u|^{p-2}=0 \text {, }
$$

where $u$ is a function on $\pi \times \mathbb{R}$ on $\pi \times I(I=$ an interval $[0, \tau])$ with an initial condition

$$
u(x, 0)=\varphi(x)
$$

where $\varphi$ is a periodic function of $x$. Here $\pi$ stands for the circle, i.e. $\mathbb{R} / \mathbb{Z}$.

In the nonperiodic case (replacing $\pi$ by $\mathbb{R}$ ), the Cauchy problem for (1.1)(1.2) is well understood (see for instance [G-V]). One has a local solution (in the generalized sense) for (1.1) if $p-2 \leqq \frac{4}{1-2 s}$ and data $\varphi \in H^{s}(\mathbb{R}), s \geqq 0$. The exponent $\frac{4}{1-2 s}$ is called $H^{s}$-critical (in 1 space dimension). If $p \geqq 6$, there is even for smooth data a possible blow up. In this discussion, the existence result is in fact a global (or local) wellposedness theorem, in the sense of uniqueness and regularity.

In [B1], we have developed a parallel theory in the periodic case, although incomplete so far. The following facts are shown in [B1].

Theorem 1. $(p=4)$ The cauchy problem ${ }^{1}$

\footnotetext{
1 The result holds both in focusing and defocusing case (with same proof).
} 


$$
\left\{\begin{array}{l}
i u_{t}+u_{x x} \pm u|u|^{2}=0 \\
u(x, 0)=\varphi(x)
\end{array}\right.
$$

is globally wellposed for data $\varphi \in H^{s}(\pi), s \geqq 0$ and the solution $u$ is in $C(\mathbb{R}$, $\left.H^{s}(\pi)\right)$. If $u, v$ are the solutions corresponding to data $\varphi, \psi \in H^{s}$, there is the regularity estimate

$$
\|u(t)-v(t)\|_{H^{s}} \leqq C^{|t|}\|\varphi-\psi\|_{s},
$$

where $C$ depends on the $L^{2}$-size of the data, i.e.

$$
C=C\left(\|\varphi\|_{2},\|\varphi\|_{2}\right)
$$

Theorem 2. $(p>4)$ The cauchy problem ${ }^{2}$

$$
\left\{\begin{array}{l}
i u_{t}+u_{x x}+u|u|^{p-2}=0 \\
u(x, 0)=\varphi(x)
\end{array}\right.
$$

is locally wellposed on a time interval $[0, \tau]$ for data $\varphi \in H^{s}, s$ satisfying

$$
s>0 \text { for } p \leqq 6 \text { and } s>s_{*}, p-2=\frac{4}{1-2 s_{*}} \text { for } p>6
$$

where $\tau=\tau\left(\|\varphi\|_{H^{s}}\right)>0$. Again the solution $u$ is in $C\left([0, \tau], H^{s}\right)$ and depends in a Lipschitz way on $\varphi$. The dependence of $\tau$ on $\|\varphi\|_{s}^{-1}$ is power-like (see (2.24), (2.25) below).

Our investigations in the periodic case originate from the work of LebowitzRose-Speer [L-R-S] on the statistical mechanics aspects of the flow. In fact, we will complete here some of their investigations by proving the invariance of the measure they introduced on a certain (natural) statistical ensemble. In the case $p=4$, one basically has to combine their work with Th.1 on the existence of the flow. For $6 \geqq p>4$, there is a problem, in the sense that the flow is only locally defined, on a time interval depending on the size of the data in $H^{s}$-norm $(s>0$ arbitrarily chosen). For $1>s>0$, there is no apriori bound on $\|u(t)\|_{H^{s}}$. It is possible however to combine the local existence result and the invariant measure ideas to construct both the flow on the statistical ensemble and the measure. This gives in particular rise to global solutions of (1.1), (1.2) for $4<p \leqq 6$ and data $\varphi \in H^{s}(\pi), s<1^{(2)}$. More precisely, for almost all $\omega$, the random Fourier series

$$
\varphi_{a, \omega}(x)=a+\sum_{\substack{j \in \mathbb{Z} \\ j \neq 0}} \frac{g_{j}(\omega)}{j} e^{2 \pi i j x},
$$

where $a \in \mathbb{C}$ and $\left\{g_{j}(\omega)\right\}$ independent $L^{2}$-normalized Gaussians yields for $p<6$ a "good" data, meaning that (1.1), (1.2) with $\varphi=\varphi_{a, \omega}$ is globally wellposed. If $p=6$, the same statement is true, provided one imposes a restriction

$$
\left\|\varphi_{a, \omega}\right\|_{2}<c_{1}
$$

where $c_{1}$ is a certain positive constant. This restriction is linked with the discussion in [LRS] on the definition of the measure.

\footnotetext{
2 The condition $p \leqq 6$ is only needed for the normalizability of the Gibbs measure and may be dropped in the non-focusing case.
} 
The natural conserved quantities for the NLSE (1.1) are the $L^{2}$-norm:

$$
N(u(t)), \text { where } N(\phi)=\int_{\pi}|\phi(x)|^{2} d x .
$$

\section{Hamiltonian:}

$$
H(u(t)), \text { where }(\phi)=\frac{1}{2} \int_{\pi}\left|\phi^{\prime}(x)\right|^{2} d x-\frac{1}{p} \int_{\pi}|\phi(x)|^{p} d x .
$$

The (unnormalized) measure studied in [LRS] is formally given by

$$
\exp [-\beta H(\phi)] \prod_{x \in \pi} d \phi(x)
$$

where $\beta>0$ is a parameter. In order to make this measure normalizable, one imposes a bound on the $L^{2}$-norm of $\phi$, i.e.

$$
N(\phi)<B
$$

For $p<6, B$ may be chosen arbitrary, for $p=6, B$ is a specific constant. From the $L^{2}$-conservation, a cutoff $(1.11)$ is clearly acceptable. The conservation of the Hamiltonian (1.9) implies the formal invariance of (1.10). Our main problem is to make this rigourous. One approach is to replace the NLSE by a discrete system (ODE) (cf. [ML-S, Zhl])

$$
i \dot{q}_{n}+\frac{q_{n+1}+q_{n-1}-2 q_{n}}{h^{2}}+q_{n}\left|q_{n}\right|^{p-2}=0
$$

or

$$
i \dot{q}_{n}+\frac{q_{n+1}+q_{n-1}-2 q_{n}}{h^{2}}+\frac{1}{2}\left(q_{n+1}+q_{n-1}\right)\left|q_{n}\right|^{p-2}=0,
$$

where $n=0, \ldots, N-1 \in \mathbb{Z}_{N} \equiv \mathbb{Z} / N \mathbb{Z}, h=\frac{2 \pi}{N}$ and thus the unknown function $u$ from (1.1) becomes now a vector valued time function $q=\left(q_{0}, q_{1}, \cdots, q_{N-1}\right)$. Both (1.12), (1.13) are Hamiltonian. In case of (1.12) the Hamiltonian is given by

$$
H(q)=\frac{1}{2 h} \sum\left|q_{n+1}-q_{n}\right|^{2}-\frac{1}{p} h \sum\left|q_{n}\right|^{p}
$$

and one defines a statistical ensemble by normalizing the density $e^{-\beta H(q)}$ on a suitable ball $\left[\left(\sum\left|q_{n}\right|^{2}\right)^{\frac{1}{2}}\right]$ in $\mathbb{C}^{N}$.

Up to some technical difficulties due to replacement of the circle $\pi$ by the cyclic group $\mathbb{Z}_{N}$, the estimates from [B1] carry over to the discrete case (with regularity bounds independent of $N$ ) and on finite time intervals the Cauchy problem (1.1), (1.2) is essentially the limit of (1.12) for $N \rightarrow \infty$. In the case $p>4$, the invariance considerations are exploited on the discrete level to improve on the existence results of [B1] for individual functions. Passing to the limit for $N \rightarrow \infty$ in a proper way (using the Fourier transform) yields both global solutions almost surely in the statistical ensemble and an invariant measure.

In carrying out this program, we will rather follow the method used by Zhidkov (See [Zh2]) that will avoid harmonic analysis on cyclic groups. Fix $N$ and define 


$$
P_{N^{\phi}}=\sum_{|n| \leqq N} \widehat{\phi}(n) e^{2 \pi i n x}
$$

the $N^{\text {th }}$ partial sum operator in the trigonometric system. Consider the equation

$$
i u_{t}^{N}+u_{x x}^{N}+P_{N}\left(u^{N}\left|u^{N}\right|^{p-2}\right)=0
$$

where $u^{N}$ has the form

$$
u^{N}(x, t)=\sum_{|n| \leqq N} a_{n}(t) e^{2 \pi i n x}
$$

We identify $u^{N}$ and $\bar{a}=\left(a_{n}\right)_{|n| \leqq N}$ through (1.17). Equation (1.16) is Hamiltonian since it may be written as $\left(\frac{\partial}{\partial \bar{a}}\right.$ denoting the $\bar{\partial}$ - differential of the functional)

$$
\frac{d a}{d t}=-i \frac{\partial}{\partial \bar{a}} H
$$

where

$$
H(a)=2 \pi^{2} \sum_{|n| \leqq N} n^{2}\left|a_{n}\right|^{2}-\frac{1}{p} \iint_{\pi}\left|\sum_{|n| \leqq N} a_{n} e^{2 \pi i n x}\right|^{p} d x .
$$

Thus (1.16) will be our finite dimensional models. Zhidkov uses this technique for nonlinear wave equations $u_{t t}-u_{x x}+f(x, u)=0$, where $f$ is real continuously differentiable with size estimates

$$
\frac{|f(x, u)|}{\left(1+u^{2}\right)^{1 / 2}}+\left|\partial_{u} f(x, u)\right|<A .
$$

In fact, it is observed in [Zh2] that the NLSE may be treated as well by this method, up to a sufficient knowledge of the Cauchy problem. For $p=4$, this information is given by Theorem 1 above. For $4<p \leqq 6$, the space $L^{2}(\pi)$ has to be replaced by $H^{s}(\pi)$ for some $s>0$, there is the difficulty that Theorem 2 only yields local solutions. The main steps in what follows may be summarized as

(i) The Cauchy problem for Eq.(1.6) (with bounds independent of $N$ ) and the convergence of solutions of (1.16) to solutions of (1.1).

(ii) Construction of invariant measures for (1.16). Use of the invariant measure to piece local solutions to global solutions on large subsets of the statistical ensemble corresponding to (1.16).

(iii) Global wellposedness of (1.1) for almost all data $\phi$ in the Wiener space with suitable $L^{2}$ cutoff.

(iv) Invariance of the limit measure.

As in [L-R-S], this limit measure is just a certain density on the space of periodic Wiener paths. The method of [B1] is very flexible and permits us to deal with other nonlinearities than those appearing in (1.1), including a nonlocal nonlinear expression $P_{N}\left(u|u|^{p-2}\right)$ appearing in (1.16). There is essentially no change in the argument.

We mention also preprints by H. McKean and K. Vaninsky ([McK-V1,2) where invariant measures are constructed by purely probabilistic techniques. 


\section{Estimates Related to the Cauchy Problem}

In this section we will summarize the 1-dimensional results from [B1] and elaborate on certain aspects needed later on. We will indicate the main ideas for proofs which appear in detail in [B1].

Consider a NLSE

$$
i u_{t}+u_{x x}+\Gamma(u)=0,
$$

where $u$ is a space periodic function of $x$ and $\Gamma(u)$ a function of $u$ (with not necessarily local dependence). For proving local wellposedness results, replace (2.1) by the equivalent integral equation

$$
u(x, t)=S(t) \phi+i \int_{0}^{t} S(t-s) \Gamma(u)(s) d s
$$

where $u(x, 0)=\phi(x)$ and $S(t)$ is the unitary group associated to the linear equation. Thus

$$
S(t) \psi(x)=\sum_{n \in \mathbb{Z}} \widehat{\psi}(n) e^{i\left(n x-n^{2} t\right)}
$$

is the solution to

$$
\left\{\begin{array}{l}
i u_{t}+u_{x x}=0 \\
u(x, 0)=\psi(x)
\end{array}\right.
$$

The method consists in applying Picard's fixpoint theorem to obtain a local solution to $(2.2)$, choosing the time interval $t \in[0, \tau]$ sufficiently small depending on the size of the data. The loss of integrability due to the nonlinear term $\Gamma(u)$ is compensated by certain "regularizing" effects of $S(t)$ (as in the $\mathbb{R}$-case, where one uses Strichartz's inequality).

There is an $L^{4}$-inequality

$$
\|S(t) \psi\|_{L^{4}(\pi \times[0,1])} \leqq C\|\psi\|_{L^{2}(\pi)}
$$

and also

$$
\left\|\sum_{n} \int d \lambda a(n, \lambda) e^{i(n x+\lambda t)}\right\|_{L^{4}(\pi \times[0,1])} \leqq C\left(\sum_{n} \int d \lambda\left(1+\left|\lambda+n^{2}\right|\right)^{\frac{3}{4}}|a(n, \lambda)|^{2}\right)^{\frac{1}{2}} .
$$

One has the "almost" $L^{6}$-inequality

$$
\|S(t) \psi\|_{L^{6}(\pi \times[0,1])} \ll N^{\varepsilon}\|\psi\|_{L^{2}}(\pi)
$$

if $\operatorname{supp} \widehat{\psi} \subset[-N, N] .^{3}$

In previous inequalities $x \in \pi, t \in[0,1]$ (they are local in time). The (necessary) presence of the $N^{\varepsilon}$-factor in (2.7) is one of the differences between the periodic and $\mathbb{R}$-case. Rewriting the integral in (2.2) using Fourier transform, one gets

$$
\sum_{n} \int d \lambda \widehat{\Gamma(u)}(n, \lambda) e^{i n x} \frac{e^{i \lambda t}-e^{-i n^{2} t}}{\lambda+n^{2}}
$$

3 The symbol "«" has the usual meaning: For each $\varepsilon>0$, there is a constant $C_{\varepsilon}$ for which the inequality holds. It would be very interesting to decide whether the bound is logarithmic in $N$. 
There is no problem with possible smallness of the denominator $\lambda+n^{2}$ because $t \in[0,1]$. Consider the case of a cubic nonlinearity, i.e.

$$
\Gamma(u)=u|u|^{2} \quad(\text { as in Theorem } 1)
$$

or

$$
\Gamma(u)=P_{N}\left(u|u|^{2}\right) \quad(\text { cf.(1.16) with } p=4) .
$$

We use $L^{4}(\pi \times[0,1])$ or $L^{4}(\pi \times[0, \tau])$ to perform the fixpoint argument. Inequality 2.5) takes care of the $S(t) \phi$ term. For the integral term (2.8), there are essentially the contributions

$$
\sum_{n} \int_{\left|\lambda+n^{2}\right|>1} d \lambda \frac{\widehat{\Gamma(u)}(n, \lambda)}{\lambda+n^{2}} e^{i(n x+\lambda t)}
$$

and

$$
\sum_{n}\left[\int_{\left|\lambda+n^{2}\right|>1} d \lambda \frac{\widehat{\Gamma(u)}(n, \lambda)}{\lambda+n^{2}}\right] e^{i\left(n x-n^{2} t\right)}
$$

which are estimated using (2.6) and the dual inequality. Thus

$$
\begin{aligned}
&\|(2.11)\|_{L^{4}(\pi \times[0,1])} \leqq C\left(\sum_{n} \int d \lambda \frac{|\widehat{\Gamma(u)}(n, \lambda)|^{2}}{1+\left|\lambda+n^{2}\right|^{\frac{5}{4}}}\right)^{\frac{1}{2}} \\
& \leqq C\left(\sum_{n} \int d \lambda \frac{|\widehat{\Gamma(u)}(n, \lambda)|^{2}}{1+\left|\lambda+n^{2}\right|^{\frac{3}{4}}}\right)^{\frac{1}{2}} \\
& \leqq C\|\Gamma(u)\|_{L^{\frac{4}{3}}(\pi \times[0,1])}
\end{aligned}
$$

and by Hölder's inequality

$$
\begin{aligned}
&\|(2.12)\|_{L^{4}(\pi \times[0,1])} \leqq C\left[\sum_{n}\left(\int d \lambda \frac{|\widehat{\Gamma(u)}(n, \lambda)|}{1+\left|\lambda+n^{2}\right|}\right)^{2}\right]^{\frac{1}{2}} \\
& \leqq C\left(\sum_{n} \int d \lambda \frac{|\widehat{\Gamma(u)}(n, \lambda)|^{2}}{1+\left|\lambda+n^{2}\right|^{\frac{3}{4}}}\right)^{\frac{1}{2}} \leqq C\|\Gamma(u)\|_{\frac{4}{3}} .
\end{aligned}
$$

Since $P_{N}$ is bounded on $L^{P}(\pi)(1<p<\infty$, independently of $N)$, in both cases (2.9), (2.10) there is the estimate $\|\Gamma(u)\|_{\frac{4}{3}} \leqq C\|u\|_{4}^{3}$.

In fact, from the extra saving on the power of the denominator $\lambda+n^{2}$, one may obtain a bound $C \cdot \tau^{\delta} \cdot\|u\|_{4}^{3}$ replacing the time interval $[0,1]$ by a small interval $[0,1]$. Here $\delta>0$ is some constant. Similarly, one shows that

$$
\begin{gathered}
\left\|\int_{0}^{t} S(t-s) \Gamma(u)(s) d s-\int_{0}^{t} S(t-s) \Gamma(v)(s) d s\right\|_{L^{4}(\pi \times[0,1])} \\
\leqq C \cdot \tau^{\delta}\left(\|u\|_{4}+\|v\|_{4}\right)^{2}\|u-v\|_{4}
\end{gathered}
$$


Hence, for $\tau=\tau\left(\|\phi\|_{s}\right)<\frac{1}{\left(\|\phi\|_{s}\right)^{C}}$ small enough, the contraction principle is easily verified. Observe from the preceding that we have bounded

$$
\begin{aligned}
\|u\|_{L_{t}^{\infty} L_{x}^{2}} & \leqq\|\phi\|_{2}+\left[\sum_{n}\left(\int d \lambda \frac{|\widehat{\Gamma(u)}(n, \lambda)|}{1+\left|\lambda+n^{2}\right|}\right)^{2}\right]^{\frac{1}{2}} \\
& \leqq\|\phi\|_{2}+C\|u\|_{4}^{3},
\end{aligned}
$$

and in fact shown that $u \in C\left([0, \tau] ; L^{2}(\pi)\right)$.

If $u, v$ are the local solutions corresponding to data $\phi, \psi$, one has for $t \in[0, \tau]$,

$$
\|u(t)-v(t)\|_{L^{2}(\pi)} \leqq 2\|\phi-\psi\|_{L^{2}(\pi)},
$$

which is the regularity property. The discussion of the cubic case for $H^{s}, s>0$, instead of $L^{2}$ is similar. One just introduces an extra $\partial_{x}^{(s)}$ in the preceding. The time interval $[0, \tau]$ however only depends on $\|\phi\|_{2}$, conserved under the flow. From this conservation, the local wellposedness theorem leads to a global result. One has the regularity estimate

$$
\|u(t)-v(t) H\|_{H^{s}(\pi)} \leqq C^{|t|}\|\phi-\psi\|_{H^{s}(\pi)},
$$

where $C=C\left(\|\phi\|_{2},\|\phi\|_{2}\right)$.

The analysis for $p>4$ in Theorem 2 or (1.16) is more complicated, mainly due to the presence of a factor $N^{\varepsilon}$ in (2.7). Here we are unable to deal with general $L^{2}$-data. The local result requires $\phi \in H^{s}(\pi)$. Any $s>0$ will do for $p \leqq 6$. The norm used to apply a contraction principle is expressed using Fourier transform

$$
\|u \mid\|_{s}=\sup _{K}(1+K)^{\frac{1}{2}}\left(\sum_{n \in \mathbb{Z}}(1+|n|)^{2 s} \int_{K \leqq\left|\lambda+n^{2}\right| \leqq 2 K+1}|\widehat{u}(n, \lambda)|^{2}\right)^{\frac{1}{2}},
$$

where we let $K$ take dyadic values. We consider functions $u$ on $\pi \times[0, \tau]$ given by a Fourier series

$$
u(x, t)=\sum_{n} \int d \lambda \widehat{u}(n, \lambda) e^{i(n x+\lambda t)}
$$

on $\pi \times[0, \tau]$. There is no uniqueness and (2.19) is obtained as infimum over representations (2.20). To make estimates on (2.8), one considers a Littlewood-Paley decomposition of $u$ w.r.t. the $x$-variable, thus

$$
u=\sum_{M \text { dyadic }} u_{M} ; u_{M}=P_{M} u-P_{\frac{M}{2}} u
$$

and writes the nonlinearity $u|u|^{p-2}$ as

$$
u|u|^{p-2}=\sum_{\substack{M_{1}>M_{2} \\ \text { dyadic }}} \tilde{u}_{M_{1}} \tilde{u}_{M_{2}} H\left(P_{10 M_{2}} u\right)
$$

with $\tilde{u}=u$ or $\tilde{u}=\bar{u}$. If $p=$ even integer, for instance $p=6$, this is purely algebraic. For other values of $p$, one uses the fact that $z|z|^{p-2}$ is sufficiently differentiable. The main idea is that the first factor $u_{M_{1}}$ takes care of the $M^{s}$ factor 
appearing in the definition of the norm (2.19) and the $M_{2}^{-s}$ associated with the second factor $u_{M_{2}}$ takes care of $N^{\varepsilon}$-factors appearing when estimating the norm (2.19) of the summants in (2.22) using (2.7). See [B1] for details.

If $\phi \in H^{s}(\pi), s>0$, one gets a local solution for $p \leqq 6$. The time interval $\tau$ of existence depends on $s$ and $\|\phi\|_{s}$. On that interval $[0, \tau]$, the solution $u$ is in $\left.C\left([0, \tau] ; H^{s} \pi\right)\right)$. If $u, v$ are the solutions with data $\phi, \psi$, there is the regularity estimate

$$
\|u(t)-v(t)\|_{H^{s_{1}}} \leqq 2\|\phi-\psi\|_{H^{s_{1}}} \text { for all } s_{1},
$$

provided $t<\tau=\tau\left(s_{0},\|\phi\|_{H} s_{0}+\|\psi\|_{H}\right)$, for some $s_{0}>0$.

It is of importance to notice that the size $\tau$ of the time interval depends on the \|\|$_{s}$-size of the data as a power. More precisely, if $p<6$ one has

$$
\tau>C(p, s) \frac{1}{\left(1+\|\phi\|_{s}\right)^{C(p)}},
$$

and for $p=6$

$$
\tau>\frac{C(s)}{\left(1+\|\phi\|_{s}\right)^{C(s)}} .
$$

The problem to build global solutions from this local result is that $\|u(t)\|_{s}$ is not conserved under the flow of the NLSE and there is no apriori bound for $0<s<1$. We will show in this paper how the invariant measure yields a substitute for a conservation law, considering the IVP for a set of data rather than a single function.

Equation (1.16) corresponds to a vector valued ODE in $\left(a_{n}\right)_{|n| \leqq N}$. From the apriori bound (1.18), the solution has to remain bounded and hence is defined for all time. On the other hand, the same argument mentioned above for the NLSE (1.1) permits to prove a local wellposedness theorem for (1.16) with bounds independent of $N$, for an initial data bounded in some $H^{s}, s>0$, thus

$$
\left(\sum_{|n| \leqq N}(1+|n|)^{2 s}\left|a_{n}(0)\right|\right)^{\frac{1}{2}}<C,
$$

where the size of the time interval $[0, \tau]$ depends on (2.24). Using the invariant measure argument, we will improve on this statement for certain data. The next problem is then to compare solutions of (1.16) and (1.1). The main result is given by

Lemma 2.27. Let $p \leqq 6, s>0, \varphi \in H^{s}(\pi),\|\varphi\|_{s}<A$ and $N$ a large integer. Assume the solution of

$$
\left\{\begin{array}{l}
i v_{t}+v_{x x}+P_{N}\left(v \cdot|v|^{p-2}\right)=0 \\
v(x, 0)=\sum_{|n| \leqq N} \widehat{\varphi}(n) e^{2 \pi i n x}
\end{array}\right.
$$

(we denote $u^{N}$ in (1.16) by $v$ ) satisfies

$$
\|v(t)\|_{s}<A \text { for } t \leqq T .
$$


Then the IVP (1.1)

$$
\left\{\begin{array}{l}
i u_{t}+u_{x x}+u|u|^{p-2}=0 \\
u(x, 0)=\varphi(x)
\end{array}\right.
$$

is wellposed on $[0, T]$ and there is the approximation for $t<T$ and $0<s_{1}<s$,

$$
\|u(t)-v(t)\|_{s_{1}}<\exp \left[C(p, s)(1+A)^{C_{1}(p, s)} \cdot T\right] \cdot N^{s_{1}-s},
$$

provided the expression on the right in (2.29) remains $<1$. The constant $C_{1}(p, s)$ does not depend on $s$ for $p<6$.

Proof. The proof follows the methods of [B1]. Fix $0<s_{1}<s$ and denote $\tau$ the time interval given by (2.24), (2.25) for a data bounded by $A+1$ in $H^{s_{1}}$-norm. The information on $[0, T]$ will be obtained by piecing together estimates on intervals of length $\tau$. One has the bound

$$
\|u(0)-v(0)\|_{s_{1}}=\left(\sum_{|n|>N}|n|^{2 s_{1}}|\widehat{\varphi}(n)|^{2}\right)^{\frac{1}{2}}<N^{s_{1}-s} A
$$

from assumption. Assume for $t \leqq t_{0}$ we obtained

$$
\|u(t)-v(t)\|_{s_{1}}<\delta<1 .
$$

Thus $\left\|u\left(t_{0}\right)\right\|_{s_{1}} \leqq\left\|v\left(t_{0}\right)\right\|_{s_{1}}+\delta<A+1$. From the local regularity theorem for the NLSE, the IVP

$$
\begin{aligned}
& \left\{\begin{array}{l}
i u_{t}+u_{x x}+u|u|^{p-2}=0 \\
\text { data } u\left(t_{0}\right) \text { at } t=t_{0}
\end{array}\right. \\
& \left\{\begin{array}{l}
i u_{t}^{\prime}+u_{x x}^{\prime}+u^{\prime}\left|u^{\prime}\right|^{p-2}=0 \\
u^{\prime}\left(t_{0}\right)=v\left(t_{0}\right)
\end{array}\right.
\end{aligned}
$$

are wellposed for $t \in\left[t_{0}, t_{0}+\tau\right]$. Moreover, from the regularity, there is the approximation for $t \leqq t_{0}+\tau$,

$$
\left\|u(t)-u^{\prime}(t)\right\|_{s_{1}} \leqq 2\left\|u\left(t_{0}\right)-u^{\prime}\left(t_{0}\right)\right\|_{s_{1}}<2 \delta
$$

by (2.31). Next, we need to compare $u^{\prime}(t)$ and $v(t)$ on $\left[t_{0}, t_{0}+\tau\right]$. Since the initial conditions are the same, the integral equation (2.2) gives

$$
u^{\prime}(t)-v(t)=i \int_{0}^{t} s(t-\tau) \Gamma(\tau) d \tau, \quad \text { where } \quad \Gamma=u^{\prime}\left|u^{\prime}\right|^{p-2}-P_{N}\left(v|v|^{p-2}\right)
$$

Split the expression $\Gamma$ as

$$
\Gamma=u^{\prime}\left|u^{\prime}\right|^{p-2}-P_{N}\left(u^{\prime}\left|u^{\prime}\right|^{p-2}\right)+P_{N}\left(u^{\prime}\left|u^{\prime}\right|^{p-2}-v|v|^{p-2}\right) .
$$

Denote $\left|\|\mid\|_{s_{1}}\right.$ the Fourier restriction norm given by (2.19) relative to the interval $\left[t_{0}, t_{o}+\tau\right]$. We estimate $\left\|u^{\prime}-v\right\|_{s_{1}}$ from the right side of (2.35).

From the estimates of, [B1], the first term of (2.36) contributes essentially as

$$
C \cdot \tau^{\delta}\|\| u^{\prime}-P_{N_{1}} u^{\prime}\|\|_{s_{1}}\left\|u^{\prime}\right\|_{s_{1}}^{p-2},
$$


where $N_{1} \sim N$. For the second term in (2.36), one gets

$$
C \tau^{\delta}\|\| u^{\prime}-v \|_{s_{1}}\left(\|\| u^{\prime}\left\|_{s_{1}}+\right\| v v \|_{s_{1}}\right)^{p-2} .
$$

From the local theorem one has

$$
\begin{aligned}
\left\|u^{\prime}\right\|_{s_{1}} & \leqq C\left\|u^{\prime}\left(t_{0}\right)\right\|_{s_{1}}=C\left\|v\left(t_{0}\right)\right\|_{s_{1}}<C A, \\
\|v v\|_{s_{1}} & \leqq C\left\|v\left(t_{0}\right)\right\|_{s_{1}}<C A,
\end{aligned}
$$

and the choice of $\tau$ yields

$$
\left\|u^{\prime}-v\right\|_{s_{1}}<\left\|u^{\prime}-P_{N_{1}} u^{\prime}\right\|_{s_{1}} .
$$

Considering $s>s_{1}$ rather than $s_{1}$, the local result also implies

$$
\left\|u^{\prime}\right\|_{s} \leqq C\left\|u^{\prime}\left(t_{0}\right)\right\|_{s}=C\left\|v\left(t_{0}\right)\right\|_{s}<C A
$$

from the assumption in the theorem. From (2.41) and definition (2.19), it follows that

$$
\left\|u^{\prime}-P_{N_{1}} u^{\prime}\right\|_{s_{1}} \leqq C A N_{1}^{s_{1}-s}=C A N^{s_{1}-s} .
$$

For $t \in\left[t_{0}, t_{0}+\tau\right]$, estimate (cf. (2.16))

$$
\begin{aligned}
\left\|u^{\prime}(t)-v(t) \mid\right\|_{s_{1}} & \leqq C\left(\sum|n|^{2 s_{1}}\left|\int \frac{|\widehat{\Gamma}(n, \lambda)|}{1+\left|\lambda+n^{2}\right|} d \lambda\right|^{2}\right)^{\frac{1}{2}} \\
& \leqq C\left(\left\|\left|\left\|u^{\prime}-v\right\|_{s_{1}}+\left\|\mid u^{\prime}-P_{N_{1}} u^{\prime}\right\|_{s_{1}}\right)<C A N^{s_{1}-s}\right.\right.
\end{aligned}
$$

applying (2.40), (2.42). Combined with (2.34), this yields

$$
\|u(t)-v(t)\|_{s_{1}}<2 \delta+C A N^{s_{1}-s} .
$$

Break the interval $[0, T]$ up in subintervals of length $\tau$. For $t_{j}=j \tau, j=0, \ldots, \frac{T}{\tau}$, one gets by $(2.30),(2.44)$,

$$
\left\{\begin{array}{l}
\left\|u\left(t_{j}\right)-v\left(t_{j}\right)\right\| \equiv \delta_{j+1}<2 \delta_{j}+C A N^{s_{1}-s} \\
\delta_{0}<N^{s_{1}-s} A
\end{array}\right.
$$

hence

$$
\delta_{j}<C^{j+1} A N^{s_{1}-s} .
$$

Here $J<\frac{T}{\tau}$, where $\tau>C(p, s) \frac{1}{(1+A)^{C_{1}(p, s)}}$, according to (2.24), (2.25). Estimate (2.29) follows, provided this quantity in 1-bounded. This proves the lemma.

\section{Remark.}

(i) Of course in the main application of (2.29), the parameters $p, s, A, T$ will be fixed and $N \rightarrow \infty$ so that the precise form on the inequality is not important there.

(ii) A similar result (with the appropriate interpretation) may be shown when comparing solutions of the ODE (1.12) and solutions of (1.1). 


\section{Invariant Measure for the Modified Equation (1.16)}

Recall (1.16)

$$
i u_{t}+u_{x x}+P_{N}\left(u|u|^{p-2}\right)=0
$$

where

$$
u(x, t)=\sum_{|n| \leqq N} a_{n}(t) e^{2 \pi i n x} .
$$

There is a conservation of

$$
\left(\sum_{|n| \leqq N}\left|a_{n}\right|^{2}\right)^{\frac{1}{2}}
$$

and

$$
H(a)=2 \pi^{2} \sum_{|n| \leqq N} n^{2}\left|a_{n}\right|^{2}-\frac{1}{p} \int\left|\sum_{|n| \leqq N} a_{n} e^{2 \pi i n x}\right|^{p} d x .
$$

Equation (3.1) has the advantage on the original NLSE (1.1) that the phase space is finite dimensional $\left(\mathbb{C}^{2 N+1}\right.$ or $\left.\mathbb{R}^{2(2 N+1)}\right)$ and the flow is defined for all time. Since (3.1) is equivalent with the Hamiltonian equation

$$
\dot{a}=-i \frac{\partial H}{\partial \bar{a}},
$$

the flow preserves the Lebesgue measure on the phase space $\mathbb{R}^{2(2 N+1)} \simeq \mathbb{C}^{2 N+1}$ in which coordinates are the real and complex parts of $a=\left(a_{n}\right)_{|n| \leqq N}$.

Let $B$ be a cutoff in $L^{2}$ (to be specified) and consider the ball $\mathbb{C}^{2 N+1}$,

$$
\Omega=\Omega_{N, B}=\left\{\left(a_{n}\right)_{|n| \leqq N} \mid\left(\sum\left|a_{n}\right|^{2}\right)^{\frac{1}{2}} \leqq B\right\}
$$

invariant under the flow.

Let $\rho=\rho_{N}$ be the measure on $\mathbb{C}^{2 N}=\left[\left(a_{n}\right)_{\substack{|| n \mid ⿰ N \\ n \neq 0}}\right]$ with normalized density

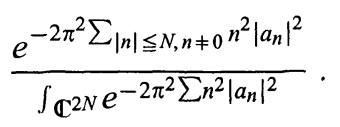

This measure is also the image measure on $\mathbb{C}^{2 N}$ under the map

$$
\omega \mapsto\left\{\frac{g_{n}(\omega)}{2 \pi n} ;|n| \leqq N, n \neq 0\right\},
$$

where the $\left\{g_{n}\right\}$ are independent equidistributed complex Gaussian random variables. The statistical ensemble will be the measure space obtained by endowing $\Omega$ with the measure ${ }^{4}$

$$
d \mu=d \mu_{N}=e^{\frac{1}{p} \int_{\pi}\left|\sum a_{n} e^{2 \pi i n x}\right|^{p} d x} \cdot\left(d a_{0} \otimes \rho_{N}\right)
$$

which, by construction, is invariant under the flow.

\footnotetext{
${ }^{4}$ Alternatively, one may redefine the equation replacing $-\Delta$ by $-\Delta+\delta, \delta>0$ to avoid the problem with the zero Fourier mode.
} 
Thus $\mu_{n}$ is a weighted Wiener measure restricted to $\Omega_{N}$. The density satisfies uniform $L^{1}\left(d \rho_{N}\right)$-estimates for $p<6$ and if $p=6$ provided the cutoff $B$ is sufficiently small. The proof of this last fact appears in [L-R-S]. We give another proof here which may be easily adjusted to other densities.

Lemma 3.10. The function $e^{\left\|\sum_{n \neq 0} \frac{g_{n}(\omega)}{n} e^{2 \pi n n x}\right\|_{p}^{p}} \chi_{\left[\left(\sum_{n \neq 0} \frac{\left|g_{n}(\omega)\right|^{2}}{n^{2}}\right)^{\frac{1}{2}}<B\right]}$ is in $L^{1}(d \omega)$ for $p<6$ and $B$ arbitrary and for $p=6, B$ sufficiently small.

(We won't go into finer details concerning best possible value of $B$ if $p=6$ here).

Proof. We estimate the probability

$$
\mathbb{P}\left[\omega \mid\left\|\sum \frac{g_{n}(\omega)}{n} e^{2 \pi i n x}\right\|_{p}>\lambda,\left(\sum \frac{\left|g_{n}(\omega)\right|^{2}}{n^{2}}\right)^{\frac{1}{2}}<B\right]
$$

Split the trigonometric system into dyadic blocks. From the second restriction and the obvious estimate

$$
\left\|\sum_{n \sim M} a_{n} e^{i n x}\right\|_{p} \leqq M^{\frac{1}{2}-\frac{1}{p}}\left\|\sum a_{n} e^{i n x}\right\|_{2}(2 \leqq p \leqq \infty),
$$

the property $\left\|\Sigma \frac{g_{n}(\omega)}{n} e^{i n x}\right\|_{p}>\lambda$ yields some

$$
M>M_{0} \equiv\left(\frac{\lambda}{B}\right)^{\frac{1}{\frac{1}{2}-\frac{1}{p}}}
$$

such that

$$
\left\|\sum_{n \sim M} g_{n}(\omega) e^{i n x}\right\|_{p}>\sigma_{M} M \lambda
$$

Here $\left(\sigma_{M}\right)_{M>M_{0}}$ is a sequence of positive numbers satisfying

$$
\sum_{\substack{M>M_{0} \\ M \text { dyadic }}} \sigma_{M}<1 .
$$

To estimate the contribution for individual $M$, consider the subspace $\left[e^{i n x} \mid n \sim M\right]_{p}$ of $L^{p}(\pi)$ generated by the characters $\left(e^{i n x}\right)_{n \sim M}$ and a norming $\operatorname{set}^{5}$ of functions $\varphi \in \xi$, such that

$$
\begin{gathered}
\max _{\varphi \in \xi}|\langle f, \varphi\rangle| \geqq \frac{1}{2}\|f\|_{p} \text { for all } f \in\left[e^{i n x} \mid n \sim M\right] \text { and } \\
\|\varphi\|_{2} \leqq M^{\frac{1}{2}-\frac{1}{p}} \text { for } \varphi \in \xi \\
\qquad \log |\xi|<C M
\end{gathered}
$$

${ }^{5}$ Given a $M$-dimensional subspace $S$ of a normed space $X$, one may always find a subset $\xi$ of the unit of the dual $X^{*}$ of cardinality $\mid \xi<c M$ such that $\max _{\varphi \in \xi}|\langle f, \varphi\rangle| \geqq \frac{1}{2}\|f\|$, for all $f \in S$. In the present situation, replace $\xi$ by its orthogonal projection $P S \xi$ on $S$, for which (3.15) clearly holds, by the Hausdorff-Young inequality. 
If $\left\|\sum_{n \sim M} g_{n} e^{i n x}\right\|_{p}>\sigma_{M} M \lambda$, then $\left|\sum g_{n}(\omega) \frac{\widehat{\varphi}(n)}{\|\varphi\|_{2}}\right|>\sigma_{M} M^{\frac{1}{2}-\frac{1}{p}} \lambda$ for some $\varphi \in \xi$ (by (3.16)) and this event has probability at most

$$
\exp \left(-c \sigma_{M}^{2} M^{1+\frac{2}{p}} \lambda^{2}\right)
$$

Summing over different $M>M_{0},(3.10)$ is the most $(\lambda>1)$

$$
\sum_{M>M_{0}} e^{C M-c \sigma_{M}^{2} M^{1+\frac{2}{p}} \lambda^{2}}<e^{-c M_{0}^{1+\frac{2}{p}}} \lambda^{2}
$$

letting $\sigma_{M}=M^{-\frac{1}{p}}+\left(\frac{M_{0}}{M}\right)^{\frac{1}{2}}$ for $M>M_{0}$. Here $M_{0}$ is given by (3.13). The integrability of the function considered in the lemma requires thus that

$$
\lambda^{p}<C B^{-\frac{1+\frac{2}{p}}{\frac{1}{2}-\frac{1}{p}}} \lambda^{\frac{1+\frac{2}{2}}{2}-\frac{1}{p}}+2
$$

for $\lambda$ large enough. If $p<6$, this is satisfied for all $B$ and for $p=6$ if $B$ is sufficiently small.

Remarks. (i) The previous argument permits to reformulate the lemma with $L^{2}(d \omega)$ (or any other finite moment) instead of $L^{1}(d \omega)$ as well. This permits us to ensure that small sets for the Wiener measure are small for $\mu_{N}$ also.

(ii) In the preceding we discussed the normalization of $e^{-H(a)} \Pi d a$. One may instead have considered $e^{-\beta H(a)} \Pi d a$ for some $\beta>0$.

For $0<s<\frac{1}{2}$ and $K>1$, consider following subset of $\Omega=\Omega_{N, B}$ :

$$
\Omega^{s, K}=\left\{\bar{a} \in \Omega \mid\left\|\sum_{|n| \leqq N} a_{n} e^{i n x}\right\|_{H^{s}(\pi)} \leqq K\right\}
$$

Since clearly

$$
\mathbb{P}\left[\omega \mid\left(\sum_{n \neq 0} \frac{\left|g_{n}(\omega)\right|^{2}}{\left(1+n^{2}\right)^{1-s}}\right)^{\frac{1}{2}}>K\right]<e^{-c K^{2}}
$$

one has

$$
\rho_{N}\left(\Omega \backslash \Omega^{s, K}\right)<e^{-c K^{2}} \text { and } \mu_{N}\left(\Omega \backslash \Omega^{s, K}\right)<e^{-c K^{2}}
$$

by previous remark.

We now come back to Eq. (3.1). If $p=4$, there is a regularity theorem wrt the $L^{2}$-norm (independent of $N$ ). For $4<p \leqq 6$, one has a uniform regularity result for data $\varphi \in H^{s}, s>0$ on a interval $[0, \tau]$ with $\tau=K^{-C}$ for $\|\varphi\|_{H^{s}} \leqq K$. This local property may be combined with the invariance of $\mu_{N}$ to get wellposedness on large time intervals $[0, \tau]$ for data in large subsets of $\Omega$. This is the main idea in this paper. We proceed as follows. The map $a \mapsto a(\tau)$ defined by the flow, thus

$$
u(x, \tau)=\sum_{|n| \leqq N} a_{n}(\tau) e^{i n x}
$$


where $u$ solves the IVP

$$
\left\{\begin{array}{l}
i u_{t}+u_{x x}+P_{N}\left(u|u|^{p-2}\right)=0 \\
u(x, 0)=\sum a_{n} e^{i n x}
\end{array}\right.
$$

is a measure preserving transformation on $\Omega, \mu$ which we denote by $S$. Let $\Omega^{s, K}$ be as above and consider the subset $\Omega_{1}$ of $\Omega$ defined by

$$
\Omega_{1}=\left(\Omega^{s, K}\right) \cap S^{-1}\left(\Omega^{s, K}\right) \cap \cdots \cap S^{-\left[\frac{T}{\tau}\right]}\left(S^{s, K}\right) .
$$

Obviously, for $a \in \Omega_{1}$, there is wellposedness (with bounds independent of $N$ ) on each subinterval $[j \tau,(j+1) \tau]$ of $[0, T]$ with corresponding data $u(j \tau)$, since by construction $\|u(j \tau)\|_{s} \leqq K$. In particular, for $a \in \Omega_{1}$, the corresponding solution $u$ of (3.1) satisfies

Furthermore, the measure

$$
\|u(t)\|_{s}<2 K \text { for } t<T \text {. }
$$

$$
\mu_{N}\left(\Omega \backslash \Omega_{1}\right) \leqq \frac{T}{\tau} \mu\left(\Omega \backslash \Omega^{s, K}\right)<T K^{C} e^{-c K^{2}}
$$

by (3.23) and $S$ measure preserving. Fixing $T,(3.24)$ is small for $K \rightarrow \infty$. We proved

Lemma 3.25. Let $0<s<\frac{1}{2}, p \leqq 6, T<\infty, \delta>0$. There is a subset $\Omega_{1}$ of $\Omega$ such that $\mu\left(\Omega \backslash \Omega_{1}\right)<\delta$ and for $\overline{\bar{a}} \in \Omega_{1}$, the solution $u$ of the IVP

$$
\left\{\begin{array}{l}
i u_{t}+u_{x x}+P_{N}\left(u|u|^{p-2}\right)=0 \\
u(x, 0)=\sum_{|n| \leqq N} a_{n} e^{2 \pi i n x}
\end{array}\right.
$$

satisfies for $|t|<T$

$$
\|u(t)\|_{H^{s}} \leqq C \sqrt{\log \frac{T}{\delta}}
$$

If $a, b \in \Omega_{1}$, the corresponding solutions satisfy for $|t|<T$ and any $s_{1}$

$$
\|u(t)-v(t)\|_{s_{1}} \leqq\left[C(p, s) \cdot T \cdot\left(\log \frac{T}{\delta}\right)^{C_{1}(p, s)}\right]\|u(0)-v(0)\|_{s_{1}},
$$

where $C_{1}(p, s)$ is independent of $s$ for $p<6$.

Estimate (3.28) is a consequence of (3.27) and the regularity results from [B1] mentioned in Sect. 2 (cf. (2.23)-(2.25)).

In fact, from (3.27) one has by interpolation with the $L^{2}$-bound

$$
\|u(t)\|_{2}=\|u(0)\|_{2}
$$

an improvement

$$
\|u(t)\|_{s} \leqq C\left(\log \frac{T}{\delta}\right)^{\alpha} \quad \text { for any } \alpha>s
$$

(interpolating between $L^{2}$ and $H^{\frac{1}{2}-\varepsilon}$ ). For $p<6$, the inequality (3.29) may be replaced by

$$
\|u(t)-v(t)\|_{s_{1}} \leqq \exp \left[C(p, \varepsilon) \cdot T \cdot\left(\log \frac{T}{\delta}\right)^{\varepsilon}\right]\|u(0)-v(0)\|_{s_{1}}
$$


for $a, b \in \Omega_{1}, u(0)=\sum a_{n} e^{i n x}, v(0)=\sum b_{n} e^{i n x}$.

Considering an increasing sequence of times $T_{j}=2^{j}$ and intersecting sets $\Omega_{1, j}$ obtained in (3.26) with measure $\mu\left(\Omega \backslash \Omega_{1, j}\right)<2^{-j} \delta$, for which (3.27) holds when $t<T_{j}$ one gets

Lemma 3.32. Let $0<s<\frac{1}{2}, p \leqq 6$ and $\delta>0$. There is a subset $\Omega_{1}$ of $\Omega$ such that $\mu\left(\Omega \backslash \Omega_{1}\right)<\delta$ and for $a \in \Omega_{1}$, the solution of the IVP

$$
\left\{\begin{array}{l}
i u_{t}+u_{x x}+P_{N}\left(u|u|^{p-2}\right)=0 \\
u(x, 0)=\sum_{|n| \leqq N} a_{n} e^{2 \pi i n x}
\end{array}\right.
$$

satisfies for all $t \in \mathbb{R}$

$$
\|u(t)\|_{s}<C\left(\log \frac{1+|t|}{\delta}\right)^{s+}
$$

$(s+$ denotes any number $>s)$.

\section{Flow and Invariant Measure for the NLS (1.1)}

We consider the limits $\rho$ and $\mu$ of the measures $\rho_{N}, \mu_{N}$ defined in Sect. 3 for $N \rightarrow \infty$. Thus $\rho$ is the image measure under the map

$$
\omega \mapsto \sum_{n \neq 0} \frac{g_{n}(\omega)}{2 \pi n} e^{2 \pi i n x}
$$

Since the random Fourier series $\sum_{n \neq 0} \frac{g_{n}(\omega)}{2 \pi n} e^{2 \pi i n x}$ is almost surely in $H_{0}^{s}(\pi)$ for all $s<\frac{1}{2}$, we may view $\rho$ as a measure on any $H_{0}^{s}(\pi), s<\frac{1}{2}$; Define $\mu$ by

$$
d \mu=\left(e^{\frac{1}{p}\|\phi\|_{p}^{p}} \chi_{\left[\|\phi\|_{2} \leqq B\right]}\right) \cdot\left(d a_{0} \otimes d \rho\right),
$$

where $B$ is the $L^{2}$-cutoff (arbitrary for $p<6$ and specific if $p=6$ ) and $a_{0}=\widehat{\phi}(0)$. Thus, from Lemma 3.10, $\frac{d \mu}{d \rho}$ is in $L^{1}(d \rho)$ (or $L^{2}(d \rho)$ ).

Denote $E_{N}=\left[e^{2 \pi i n x}|| n \mid \leqq N\right]$. If $U$ is an open set in $H^{s}, s<\frac{1}{2}$, one has (cf. $[\mathrm{Zh} 2])^{6}$

$$
\begin{aligned}
& \rho(U)=\lim _{n \rightarrow \infty} \rho_{N}\left(U \cap E_{N}\right), \\
& \mu(U)=\lim _{n \rightarrow \infty} \mu_{N}\left(U \cap E_{N}\right) .
\end{aligned}
$$

The NLSE (1.1) is globally wellposed $\mu$-almost everywhere. More precisely

Lemma 4.4 For $p \leqq 6, \delta>0$, the IVP

$$
\left\{\begin{array}{l}
i u_{t}+u_{x x}+u|u|^{p-2}=0 \\
u(x, 0)=\phi(x)
\end{array}\right.
$$

${ }^{6}$ For the first statement, use (4.1) and the almost sure convergence of the series in $H^{s}$ for all $s<\frac{1}{2}$, which makes the role of the tale negligible. For the second statement, use (42), where the density factor is an integrable function of the $\omega$-variable. 
is globally wellposed and

$$
\|u(t)\|_{s}<C\left(\log \frac{1+|t|}{\delta}\right)^{s+} \quad \text { for all } s<\frac{1}{2}
$$

for a set of data $\phi \in \Phi$ where $\mu\left(\Phi^{c}\right)<\delta$.

The set $\Phi$ may be taken compact in $H^{s}(\pi)$, for all $s<\frac{1}{2}$.

Proof. We combine Lemma 3.32 and the approximation Lemma 2.27. Fix first $0<$ $s_{1}<s<\frac{1}{2}, T<\infty$ and $\delta_{1}>0$. Clearly for appropriate $C(s)$ the set $\Phi_{1}$ defined by $\phi \in \Phi_{1}$ if

$$
\|\phi\|_{H^{s}} \leqq C(s)\left(\log \frac{1}{\delta_{1}}\right)^{\frac{1}{2}}
$$

satisfies $\mu\left(\Phi_{1}^{c}\right)<\delta_{1}$. Choose $N$ large and apply Lemma 3.32, giving a set $\Omega^{\prime}$ with $\mu_{N}\left(\Omega_{N} \backslash \Omega^{\prime}\right)<\delta_{1}^{2}$ and such that the solution $v$ of

$$
\left\{\begin{array}{l}
i v_{t}+v_{x x}+P_{N}\left(v|v|^{p-2}\right)=0 \\
v(x, 0)=\sum_{|n| \leqq N} a_{n} e^{2 \pi i n x}
\end{array},\right.
$$

where $\left(a_{n}\right)_{|n| \leqq N}$ in $\Omega^{\prime}$ satisfies for all $t$

$$
\|v(t)\|_{s}<C\left(\log \frac{1+|t|}{\delta_{1}}\right)^{\frac{1}{2}} .
$$

Define $\quad \Phi_{2}=\left[\varphi \mid(\widehat{\varphi}(n))_{|n| \leqq N} \in \Omega^{\prime}\right] . \quad$ Then $\quad\left(d a_{0} \otimes \rho\right)\left[\|\varphi\|_{2} \leqq B, \varphi \notin \Phi_{2}\right] \leqq$ $\left(d a_{0} \otimes \rho_{N}\right)\left[\|a\|_{2} \leqq B, a \notin \Omega^{\prime}\right] \leqq \mu_{N}\left(\Omega_{N} \backslash \Omega^{\prime}\right)<\delta_{1}^{2}$. Hence, since we assumed the density in (4.2) in $L^{2}\left(d a_{0} \otimes d \rho\right)$, also $\mu\left(\Phi_{2}^{c}\right) \leqq \delta_{1}$. Consequently, except for a set of $\mu$-measure at most $\delta_{1}$, we may ensure (4.5) and the bound (4.7) for the IVP (4.6) with data $\sum_{|n| \leqq N} \widehat{\varphi}(n) e^{2 \pi i n x}$.

Applying Lemma 2.27 with $A=C\left(\log \frac{T}{\delta_{1}}\right)^{\frac{1}{2}}$, it follows that for those $\phi$ the IVP

$$
\left\{\begin{array}{l}
i u_{t}+u_{x x}+u|u|^{p-2}=0 \\
u(x, 0)=\phi
\end{array}\right.
$$

is wellposed on $[0, T]$ and (2.29) holds, hence

$$
\|u(t)\|_{s_{1}}<2 A=C\left(\log \frac{T}{\delta_{1}}\right)^{\frac{1}{2}}
$$

for $|t|<T$, provided the right side of (2.29) is $<1$, which happens by choosing $N$ large.

Intersecting for an increasing sequence of times, one gets

$$
\|u(t)\|_{s_{1}}<C_{s_{1}}\left(\log \frac{1+|t|}{\delta_{1}}\right)^{\frac{1}{2}}
$$


for data $u(0)=\varphi$ outside a set of $\mu$-measure at most $\delta_{1}$. Finally, one intersects for a sequence $s<\frac{1}{2}$. This yields the lemma with bound $C_{s}\left(\log \frac{1+|t|}{\delta}\right)^{\frac{1}{2}}$. The improved bound is obtained as in (3.32), interpolating with the $L^{2}$ - estimate $\|u(t)\|_{2}=u(0) \|_{2} \leqq B$.

Observe that from the (local) regularity theorem (Th. 2), one may replace $\Phi$ by its closure in $H^{s}$, for any $s>0$.

Theorem The measure $\mu$ is invariant under the flow of the NLSE (1.1) $(4 \leqq$ $p \leqq 6)$.

Proof. We already showed that the NLSE is globally wellposed on a $K_{\sigma}$-set $\Omega$ (in $H^{s}$, for any $s<\frac{1}{2}$ ) of full $\mu$-measure. This set is simply the union of the compact sets for $\delta>0, A<\infty$,

$$
\begin{aligned}
K_{\delta, A} & =\left[\varphi \mid\|\varphi\|_{2} \leqq B \text { and }\|u(t)\|_{s}\right. \\
& \left.\leqq A C_{s}\left(\log \frac{1+|t|}{\delta}\right)^{s+} \text { for all } t \in \mathbb{R}, s<\frac{1}{2}\right]
\end{aligned}
$$

where $u$ is the solution of (1.1) with $u(0)=\varphi$.

The flow maps $\Omega$ to itself. We show the invariance of $\mu$ on $\Omega$. Denote $S$ the time shift $\varphi=u(0) \mapsto u(t)$ for some time $t$ and let $K$ be a compact in $\Omega$. Fix some $0<s<\frac{1}{2}$ and denote $B_{\varepsilon}$ the $\varepsilon$ - ball in $H^{s}$. Denote $S_{N}$ the flow on $\Omega_{N, B} \subset E_{N}$ considered in Sect. 3, given by Eq. (1.16) thus

$$
i u_{t}^{N}+u_{x x}^{N}+P_{N}\left(u^{N}\left|u^{N}\right|^{p-2}\right)=0
$$

for the same time shift $u^{N}(0) \mapsto u^{N}(t)$. Fix $\varepsilon>0$. From the regularity of $S_{N}$ in $H^{s}$ (independently of $N$ ) and the approximation property (2.27) for $S$ and $S_{N}$, one gets for some $\varepsilon>0$ and $N>N_{0}$,

$$
S_{N}\left(\left(K+B_{\varepsilon}\right) \cap E_{N}\right) \subset S_{N}\left(P_{N}(K)\right)+B_{\varepsilon_{1 / 2}} \subset S(K)+B_{\varepsilon_{1}},
$$

thus by (4.3), (4.12),

$$
\mu\left(S(K)+B_{\varepsilon_{1}}\right)=\lim _{N \rightarrow \infty} \mu_{n}\left(\left(S(K)+B_{\varepsilon_{1}}\right) \cap E_{N}\right) \geqq \varliminf_{N \rightarrow \infty} \mu_{N}\left(S_{N}\left(\left(K+B_{\varepsilon}\right) \cap E_{N}\right)\right) .
$$

But since $\mu_{N}$ is invariant under $S_{N}$,

$$
\mu\left(S(K)+B_{\varepsilon_{1}}\right) \geqq \lim \mu_{N}\left(\left(K+B_{\varepsilon}\right) \cap E_{N}\right)=\mu\left(K+B_{\varepsilon}\right) \geqq \mu(K) .
$$

Hence $\mu(S(K)) \geqq \mu(K)$. Since the flow is reversible, we conclude

$$
\mu(K)=\mu(S(K)),
$$

proving the theorem.

Remarks. (I) It follows from Lemma 4.4 that the Cauchy problem for $p \leqq 6$

$$
\left\{\begin{array}{l}
i u_{t}+u_{x x}+u|u|^{p-2}=0 \\
u(x, 0)=a_{0}+\sum_{n \neq 0} \frac{g_{n}(\omega)}{2 \pi n} e^{2 \pi i n x} \equiv \phi_{a_{0}, \omega}
\end{array}\right.
$$


is globally wellposed for almost all $a_{0}$ and $\omega$, subject to the restriction

$$
\left(\left|a_{0}\right|^{2}+\sum_{n \neq 0} \frac{\left|g_{n}(\omega)\right|^{2}}{4 \pi^{2} n^{2}}\right)^{\frac{1}{2}} \leqq B
$$

for a certain constant $B$, in case $p=6$ (for $p<6$, the $L^{2}$-cutoff is arbitrary and hence may be ignored in this statement).

The factor in front of $\frac{g_{n}(\omega)}{n}$ may be changed, considering the measure $e^{-\beta H(\phi)} \prod_{x} d \phi(x)$ for other values of $\beta$.

Assume $p<6$. Then the previous almost sure result also holds after conditioning

$$
P_{N_{o}}\left(\phi_{a_{0}, \omega}\right)=\psi(x)=\sum_{|n| \leqq N_{0}} \psi(n) e^{2 \pi i n x},
$$

where $\psi$ is an arbitrary trigonometric polynomial. This follows from the precise regularity statment (see(3.31)). Consider $\gamma>0$ and the set

$$
\Omega_{1}=\left[\left(a_{0}, w\right) \mid\left\|P_{N_{0}}\left(\phi_{a_{0}, \omega}\right)-\psi\right\|_{H^{1}}<\gamma\right]
$$

of measure $>c(\psi) \cdot \gamma^{4 \mu_{0}+2}=\delta$. From Lemma 4.4, there is an $\left(a_{0}, \omega\right) \in \Omega_{1}$ such that the solution $u$ of (4.15) satisfies for $s<\frac{1}{2}$,

$$
\|u(t)\|_{s}<C\left(\log \frac{1+|t|}{\delta}\right)^{s+}<C(\psi)\left(\log \frac{1+|t|}{\gamma}\right)^{s+} .
$$

Consider the initial data

$$
\phi=\psi+\left(\phi_{a_{0}, \omega}-P_{N_{0}}\left(\phi_{a_{0}, \omega}\right)\right)
$$

satisfying

$$
P_{N_{0}} \phi=\psi
$$

and

$$
\left\|\phi-\phi_{a_{0}, \omega}\right\|_{H^{\prime}}<\gamma .
$$

The Cauchy problem (4.15) for $u(x, 0)=\phi(x)$ will be wellposed on $[0, T]$ as long as, say

$$
\gamma \cdot \exp \frac{T}{\tau}<1
$$

where $\tau$ is the local regularity interval thus $\tau^{-1}=C(p, s)\left(\log \frac{T}{\gamma}\right)^{C(p)(s+)}, s>0$. Thus letting $s$ be small enough, (4. 31) may be realized for $\gamma \rightarrow 0$. This argument easily yields an almost sure property subject to conditioning (4.17), on any finite interval, from whence the claim.

(II) The existence of the invariant measure for the NLSE (1.1), $p=6$ yields a weak form of the Fermi-Pasta-Ulam recurrence phenomenon. The method followed here applies equally well to other Hamiltonian NLSE's provided the nonlinearity does not exceed the critical power (roughly speaking). On the other hand, results obtained in [B2] (section) permit to show that in this generality for smooth nonlinearity and smooth data, this recurrence property may not hold in higher derivatives, which may be unbounded in time (unlike the integrable case $i u_{t}+u_{x x}+u|u|^{2}=0$ ). 
(III) In the $1 D$-defocusing case, the $L^{2}$-cutoff and the restriction $p \leqq 6$ may be dropped in the invariant measure construction, as mentioned earlier.

(IV) The results in [B1] for one and higher dimensional NLSE do not cover negative Sobolev indices, which would be necessary to deal with the invariant measure problems in dimesions $\geqq 2$. $^{7}$

\section{Appendix. Invariant Measures and Korteweg de Vries Equations}

The method described in what precedes permits us to treat certain other equations, such as the $\mathrm{KdV}$ equation

$$
u_{t}+u_{x x x}+u u_{x}=0
$$

and modified $\mathrm{KdV}$ equation

$$
u_{t}+u_{x x x} \pm u^{2} u_{x}=0
$$

( $u$ is real and again space periodic). In general for the equation

$$
u_{t}+u_{x x x} \pm u^{k} u_{x}=0
$$

there is a hamiltonian

$$
\frac{1}{2} \int\left(\phi^{\prime}\right)^{2} \mp \frac{1}{(k+1)(k+2)} \int \phi^{k+2}=H(\phi),
$$

and (3) is equivalent to

$$
u_{t}=\partial_{x} \frac{\partial H}{\partial u} .
$$

We consider the cases $k=1,2$ (which are in fact the integrable ones). For $k=1$, there is an $L^{2}$-global wellposedness theorem and for $k=2$ a local wellposedness theorem in $H^{1 / 2}$ (see [B2]). Up to a slight adjustment for $k=2$, this regularity theory enables us to repeat the argument used for NLSE to get the invariant measure and, for $k=2$, the regularity of the flow almost everywhere on the statistical ensemble. For $k>2$, I only dispose presently of an $H^{1}$-theorem, which is insufficient in this discussion. The Gibbs measure $e^{-H(\phi)}=e^{ \pm \frac{1}{(k+1)(k+2)} \int \phi^{k+2}} \cdot e^{-\frac{1}{2} \int\left(\phi^{\prime}\right)^{2}} \prod_{x} d \phi(x)$ is under some $L^{2}$-cutoff ( $L^{2}$ is a conserved quantity) normalizable for $k=1,2$ and the normalized measure is again essentially the Wiener measure with some weight. For $k=2$ and - sign in (3), no $L^{2}$-cutoff is needed. Thus one needs at least local wellposedness results for data in $H^{s}(\mathbb{T})$, for some $s<\frac{1}{2}$, to make previous method work.

Fix again a large positive integer $N$ and consider the finite dimensional model

$$
u_{t}+u_{x x x}+P_{N}\left(u u^{k}\right)=0
$$

with

$$
P_{N} \phi=\sum_{|n| \leqq N} \hat{\phi}(n) e^{i n x}
$$

\footnotetext{
7 The author has recently extended the results to the cubic 2D-defocusing NLS $i u_{t}+\Delta u=u|u|^{2}-$ $2\left(\int|u|^{2}\right) u$ obtained by Wick ordering of the nonlinearity. The Gibbs measure constructed from the Wick ordered $|\phi|^{4}$-Hamiltonian is invariant under the (well-defined) flow of the equation [B3].
} 
and

$$
u=\sum_{|n| \leqq N} a_{n}(t) e^{2 \pi i n x}, \quad a_{n}=\bar{a}_{-n} .
$$

Again the technique from [B1] permits for $k=1$, say, to obtain uniform regularity estimates for (6) and approximation of the solution of the IVP

$$
\left\{\begin{array}{c}
u_{t}+u_{x x x}+u u_{x}=0 \\
u(0, x)=\phi(x)
\end{array}\right.
$$

from the solutions of

$$
\left\{\begin{array}{c}
v_{t}+v_{x x x}+P_{N}\left(v v_{x}\right)=0 \\
v(0, x)=P_{N} \phi(x)
\end{array}\right.
$$

when $N \rightarrow \infty$, assuming $\phi \in H^{s}, s>0$. See [B1], Sect. 7 .

In the model (6), the phase space is $2 N+1$ dimensional and the invariance of the measure follows from Liouville's theorem (the reader may find a systematic expression of these matters in a more general context in [Bid]). Thus for $k=1$, the situation is analogous to the cubic Schrödinger equation and there is essentially no extra work. For $k=2$, there is the problem again that the regularity theory is local and moreover in $H^{1 / 2}$ (see [B1], Proposition 8.45), which is borderline for the Wiener space.

The purpose of what follows is to indicate how to rework the arguement leading to Proposition 8.45 in order to gain on the $\frac{1}{2}$-Sobolev exponent. We repeat some steps from [B2], Sect. 8. Thus we consider the IVP

$$
\left\{\begin{array}{c}
\partial_{t} u+\partial_{x}^{3} u+u^{2} \partial_{x} u=0 \\
u(0, x)=\phi(x)
\end{array}\right.
$$

$u$ periodic in $x$.

Define

$$
c=\int \phi(x)^{2} d x,
$$

and consider the linear equation

$$
\partial_{t} u+\partial_{x}^{3} u+c \partial_{x} u=0
$$

The solution for initial data $u(x, 0)=\psi(x)$ may be written as

$$
u(x, t)=S_{t} \psi(x)=\sum_{n \in \mathbb{Z}} \widehat{\psi}(n) e^{i\left(n x+\left(n^{3}-c n\right) t\right)} .
$$

Consider the integral equation

$$
u(t)=S_{t} \phi+\int_{0}^{t} S(t-\tau) w(\tau) d \tau
$$

Where

$$
w=\left[-u^{2}+\int_{\mathbb{T}} u^{2}(x, t) d x\right] \partial_{x} u
$$

The IVP (10) is equivalent to the fixpoint problem naturally arising from (14), (15). Written in fourier transform, one has 


$$
u(x, t)=\sum_{n} \widehat{\phi}(n) e^{i\left(n x+\left(n^{3}-c n\right) t\right)}+\sum_{n} e^{i n x} \int \widehat{w}(n, \lambda) \frac{e^{i \lambda t}-e^{i\left(n^{3}-c n\right) t}}{\lambda-n^{3}+c n} d \lambda,
$$

where

$$
\begin{aligned}
\widehat{w}(n, \lambda)= & \frac{1}{3} \sum_{\substack{n=n_{1}+n_{2}+n_{3} \\
n_{1}+n_{2} \neq 0, n_{2}+n_{3} \neq 0, n_{3}+n_{1} \neq 0}} n \int \widehat{u}\left(n_{1}, \lambda_{1}\right) \widehat{u}\left(n_{2}, \lambda_{2}\right) \widehat{u}\left(n_{3}, \lambda-\lambda_{1}-\lambda_{2}\right) d \lambda_{1} d \lambda_{2} \\
& -n \int \widehat{u}\left(n, \lambda_{1}\right) \widehat{u}\left(n, \lambda_{2}\right) \widehat{u}\left(-n, \lambda-\lambda_{1}-\lambda_{2}\right) d \lambda_{1} d \lambda_{2} .
\end{aligned}
$$

Fix indices $0<s_{1}<\frac{1}{2}<s_{2}<1$ and consider following replacement of the norm (8.26) in [B2]:

$$
\begin{aligned}
\|u\| \|^{2}= & \|u\|_{s_{1}, s_{2}}^{2} \\
= & \sum_{n \in \mathbb{Z}}\left(1+|n|^{2 s_{1}}\right) \int d \lambda\left(1+\left|\lambda-n^{3}+c n\right|\right)|\widehat{u}(n, \lambda)|^{2} \\
& +\sum_{n}\left(1+|n|^{2 s_{1}}\right)\left(\int|\widehat{u}(n, \lambda)| d \lambda\right)^{2} \\
& +\sup _{n}\left(1+|n|^{2 s_{2}}\right)\left[\int d \lambda\left(1+\left|\lambda-n^{3}+c n\right|\right)|\widehat{u}(n, \lambda)|^{2}+\left(\int|\widehat{u}(n, \lambda)| d \lambda\right)^{2}\right] .
\end{aligned}
$$

The contribution of the first term of (16) to this norm is

$$
\|\phi\|_{H^{s_{1} \mathbb{T}}}+\sup _{n}\left(1+|n|^{s_{2}}\right)|\widehat{\phi}(n)| \text {. }
$$

Observe that for elements of our statistical ensemble, i.e. of the form $\sum \frac{g_{n}(\omega)}{n} e^{i n x}$, $\left\{g_{n}\right\}_{n>0}$ independent complex Gaussian random variables, $g_{-n}=\bar{g}_{n}$, the choice of $s_{1}, s_{2}$ implies that $(22)$ is almost surely finite, more precisely $(22)<K$ except on a set of measure $<e^{-c K^{2}}$ (as in the discussion of the NLSE).

For the second term in (16), there are following contributions to the norm:

$$
\begin{gathered}
(19) \leftrightarrow \sum_{n} \int d \lambda\left(1+|n|^{\left.2 s_{1}\right)} \frac{|\widehat{w}(n, \lambda)|^{2}}{1+\left|\lambda-n^{3}+c n\right|},\right. \\
(20) \leftrightarrow \sum_{n}\left(1+|n|^{2 s_{1}}\right)\left(\int \frac{|\widehat{w}(n, \lambda)|}{1+\left|\lambda-n^{3}+c n\right|} d \lambda\right)^{2} \\
(21) \leftrightarrow\left(1+|n|^{2 s_{2}}\right)\left\{\int d \lambda \frac{|\widehat{w}(n, \lambda)|^{2}}{1+\left|\lambda-n^{3}+c n\right|}+\left(\int \frac{|\widehat{w}(n, \lambda)|}{1+\left|\lambda-n^{3}+c n\right|}\right)^{2}\right\} .
\end{gathered}
$$

The key arithmetical fact underlying the estimates is the identity

$$
\left(n_{1}+n_{2}+n_{3}\right)^{3}-n_{1}^{3}-n_{2}^{3}-n_{3}^{3}=3\left(n_{1}+n_{2}\right)\left(n_{2}+n_{3}\right)\left(n_{3}+n_{1}\right)
$$

implying in particular that for $\lambda=\lambda_{1}+\lambda_{2}+\lambda_{3}, n=n_{1}+n_{2}+n_{3}$,

$$
\max \left(\left|\lambda-n^{3}+c n\right|,\left|\lambda_{i}-n_{i}^{3}+c n_{i}\right|(1 \leqq i \leqq 3)\right) \geqq\left|n_{1}+n_{2}\right|\left|n_{2}+n_{3}\right|\left|n_{3}+n_{1}\right| .
$$

If none of the sums $n_{1}+n_{2}, n_{2}+n_{3}, n_{3}+n_{1}$ vanishes, there are following possibilities: 
At least 2 factors in (27) are $\sim \max \left(\left|n_{1}\right|+n_{2}|+| n_{3} \mid\right)$ and hence

$$
(27) \geqq\left(\left|n_{1}\right|+\left|n_{2}\right|+\left|n_{3}\right|\right)^{2},
$$

$\left|n_{1}\right| \sim\left|n_{2}\right| \sim \mid n_{3}$ and at least 1 factor in (27) is $\sim \max \left(\left|n_{1}\right|, n_{2}|,| n_{3} \mid\right)$, hence

$$
(27) \geqq\left|n_{1}\right|+\left|n_{2}\right|+\left|n_{3}\right| \text {. }
$$

We will make use of following $L^{6}$-estimate (cf. (8.37) in [B1])

$$
\left\|\sum_{N_{0}<n<N_{0}+N} a_{n} e^{2 \pi i\left(n x+\left(n^{3}-c n\right) t\right)}\right\|_{L^{6}\left(\mathbb{T}^{2}\right)} \ll N^{\varepsilon}\left(\sum\left|a_{n}\right|^{2}\right)^{1 / 2} .
$$

Proof of (30). Writing $n x+\left(n^{3}-c n\right) t=n(x-c t)+n^{3} t$ and making a change of variable, this is clearly equivalent to

$$
\left\|\sum_{N_{0}<n<N_{0}+N} a_{n} e^{i\left(n x+n^{3} t\right)}\right\| \ll N^{\varepsilon}\left(\sum\left|a_{n}\right|^{2}\right)^{1 / 2} .
$$

Writing $\|f\|_{6}=\left\|f^{3}\right\|_{2}^{1 / 3}$, one needs to bound the number of integer solutions of the system

$$
\left\{\begin{array}{c}
x+y+z=a-3 N_{0} \equiv a_{1} \\
\left(N_{0}+x\right)^{3}+\left(N_{0}+y\right)^{3}+\left(N_{0}+Z\right)^{3}=b
\end{array}\right.
$$

for given $a, b$ and restricting $x, y, z$ to $\{0,1, \ldots, N\}$. This gives following equation in $x, y$ :

$$
-3 x y(x+y)+6 N_{0}\left(x^{2}+y^{2}+x y\right)+3 a_{1}(x+y)^{2}-\left(6 N_{0} a_{1}+3 a_{1}^{2}\right)(x+y)=b_{1}
$$

with

$$
b_{1}=b-3 N_{0}^{3}-3 N_{0}^{2} a_{1}+3 a_{1}^{2} N_{0}-a_{1}^{3} .
$$

Denote

$$
\left\{\begin{array}{l}
X=x+y \\
Y=x \cdot y
\end{array}\right.
$$

and rewrite (33) as

$$
-X Y+\left(a_{1}+2 N_{0}\right) X^{2}-2 N_{0} Y-a_{1}\left(2 N_{0}+a_{1}\right) X+\frac{1}{3} b_{1} .
$$

Multipy both members with $a_{1}+2 N_{0}$ and put $X_{1}=\left(a_{1}+2 N_{0}\right) X$ to get

$$
-X_{1} Y+X_{1}^{2}-2 N_{0}\left(a_{1}+2 N_{0}\right) Y-a_{1}\left(a_{1}+2 N_{0}\right) X_{1}=\frac{1}{3} b_{1}\left(a_{1}+2 N_{0}\right)
$$

which easily reduces to the form

$$
X_{2}^{2}-Y_{2}^{2}=A
$$

by one more change of variable. If $\log N_{0} \lesssim \log N$, one has $\log A \lesssim \log N$ and counting divisors gives a bound $\exp \frac{\log N}{\log \log N} \ll N^{\varepsilon}$ on the number of pairs $\left(X_{2}, Y_{2}\right)$, hence $(x, y)$ satisfying (33). If $\log N_{0} \gg \log N$, it easily follows from (33) that 


$$
6\left(x^{2}+y^{2}+x y\right)-6 a_{1}(x+y)
$$

equals $\frac{b_{1}}{N_{0}}+O\left(\frac{N^{3}}{N_{0}}\right)=\frac{b_{1}}{N_{0}}+o(1)$, hence may only take 1 value. In this case, the problem clearly reduces to lattice point counting on the oval $X^{2}+3 Y^{2}=A$ with $A \lesssim N^{2}$, from where again the $N^{\varepsilon}$ bound on the number of pairs $(x, y)$. Consequently (32) has only $\ll N^{\varepsilon}$ integer solutions, proving the lemma.

We now return to the expression (23), (24), (25), with $\widehat{w}$ given by $(17)-(18)$. consider first the contribution of $(17)$ to $\widehat{w}(n, \lambda)$.

Estimate $(23)$ by duality. Thus $\{d(n, \lambda)\}$ satisfies

$$
d(n, \lambda) \geqq 0 \text { and } \sum_{n \in \mathbb{Z}} \int d(n, \lambda)^{2} d \lambda \leqq 1
$$

and we consider the expression $\left(s=s_{1}\right)$

$$
\begin{gathered}
\sum_{\substack{n_{1}, n_{2}, n_{3} \\
n_{1}+n_{2} \neq 0, \ldots}}\left(1+\left|n_{1}+n_{2}+n_{3}\right|\right)^{1+s} \int d \lambda_{1} d \lambda_{2} d \lambda_{3} \\
\frac{d\left(n_{1}+n_{2}+n_{3}, \lambda_{1}+\lambda_{2}+\lambda_{3}\right)}{1+\left|\lambda_{1}+\lambda_{2}+\lambda_{3}-\left(n_{1}+n_{2}+n_{3}\right)^{3}-c\left(n_{1}+n_{2}+n_{3}\right)\right|^{1 / 2}} \\
\frac{c\left(n_{1}, \lambda_{1}\right)}{\left|\lambda_{1}-n_{1}^{3}+c n_{1}\right|^{1 / 2}} \frac{c\left(n_{2}, \lambda_{2}\right)}{\left|\lambda_{2}-n_{2}^{3}+c n_{2}\right|^{1 / 2}} \frac{c\left(n_{3}, \lambda_{3}\right)}{\left|\lambda_{3}-n_{3}^{3}+c n_{3}\right|^{1 / 2}}
\end{gathered}
$$

denoting

Thus from (19)

$$
c(n, \lambda)=\left(1+\left|\lambda-n^{3}+c n\right|\right)^{1 / 2}|\widehat{u}(n, \lambda)| .
$$

$$
\left[\sum_{n} \int d \lambda\left(1+|n|^{2 s}\right) c(n, \lambda)^{2}\right]^{1 / 2} \leqq\|u\| \| .
$$

Assume (28) holds. Proceeding as in [B1], Sect. 7, one of the 4 denominators in (40) takes care of a factor $\left|n_{1}\right|+\left|n_{2}\right|+\left|n_{3}\right|$, the remaining $\left(\left|n_{1}\right|+\left|n_{2}\right|+n_{3} \mid\right)^{s}$ is multiplied with $c\left(n_{i}, \lambda_{i}\right)$ if $\left|n_{i}\right|=\max \left(\left|n_{1}\right|,\left|n_{2}\right|,\left|n_{3}\right|\right)$. The new expression (40) is then formulated as the $(x, t)$-integral of 4 functions, belonging to $L^{2}, L^{6}, L^{6}, L^{6}$. This requires a cutting up of the $x$-Fourier transforms in intervals of appropriate length and the use of (30). It is the same reasoning as applied in [B1] for the NLSE in $\operatorname{dim} 1$ say, when $p=6$ and hence the $L^{6}$-bound just fails. Thus at this stage, we only need $s_{1}>0$.

Assume (29) holds. Then $\left|n_{1} \sim\right| n_{2}|\sim| n_{3} \mid$ and one of the 4 denominators in $(40)$ is at least $\left(n_{1}+\left|n_{2}+n_{3}\right|\right)^{1 / 2}$. The remaining $\left(\left|n_{1}\right|+\left|n_{2}\right|+\left|n_{3}\right|\right)^{1 / 2+s} \sim$ $\left|n_{1}\right|^{\frac{1}{6}+\frac{s}{3}}\left|n_{2}\right|^{\frac{1}{6}+\frac{s}{3}}\left|n_{3}\right|^{\frac{1}{6}+\frac{s}{3}}$ is multiplied with the $c\left(n_{i}, \lambda_{i}\right)$ factors. The $L^{2}-L^{6}-L^{6}-L^{6}$ estimate is thus applicable provided $s_{1}>\frac{1}{6}+\frac{s_{1}}{3}$, hence $s_{1}>\frac{1}{4}$. In this case, inequality (30) may be used directly, without interval partitioning, since $n_{1}\left|\sim n_{2}\right| \sim$ $\left|n_{3}\right|$.

To estimate (24), consider a system $\{d(n)\}_{n \in \mathbb{Z}}$

$$
d(n) \geqq 0 \quad \sum d(n)^{2} \leqq 1
$$

and replace in (40) the first factor by

$$
\frac{d(n)}{1+\left|\lambda-n^{3}+c n\right|} \text {. }
$$


Proceeding as in [B1] one can essentially perform the same estimates as for (40), since a denominator $\left|\lambda-n^{3}-c n\right|^{1 / 2}$ could in fact be replaced by $\left|\lambda-n^{3}-c n\right|^{1 / 2-\tau}$ (where $\tau>0$ depends on $s_{1}$ ) in the $L^{6}$-estimate.

We consider next the contribution of (17) to (25). Assume $\left|n_{1}\right|=\max \left(\left|n_{i}\right| ; i=\right.$ $1,2,3)$. If $\left|n_{1}\right| \sim \max \left(\left|n_{2}\right|, n_{3} \mid\right)$, the previous analysis is already conclusive, since in case (28), the value $s$ in (40) may be taken to be $2 s_{1}-\varepsilon$ and in case (29) provided $\frac{1}{2}+s<3 s_{1}$. Hence, letting $s_{1} \geqq \frac{9}{20}$, a value of $s_{2}>\frac{4}{5}$ is obtained. Consequently, assume $\left|n_{1}\right| \gg \max \left(\left|n_{2}\right|,\left|n_{3}\right|\right)$.

Consider a system $\{d(\lambda)\}, d(\lambda) \geqq 0, \int d(\lambda)^{2} d \lambda \leqq 1$ and estimate the square root of the first term of (25) by

$$
\begin{gathered}
\left(1+\left|n_{1}\right|\right)^{1+s_{2}} \sum_{n_{1}+n_{2}+n_{3}=n} \int d \lambda_{1} d \lambda_{2} d \lambda_{3} \\
\frac{d(\lambda)}{1+\left|\lambda-n^{3}+c n\right|^{1 / 2}} \frac{c\left(n_{1}, \lambda_{1}\right)}{1+\left|\lambda_{1}-n_{1}^{3}+c n_{1}\right|^{1 / 2}} \frac{c\left(n_{2}, \lambda_{2}\right)}{\left|\lambda_{2}-n_{2}^{3}+c n_{2}\right|^{1 / 2}} \frac{c\left(n_{3}, \lambda_{3}\right)}{\left|\lambda_{3}-n_{3}^{3}+c n_{3}\right|^{1 / 2}}
\end{gathered}
$$

where $\lambda=\lambda_{1}+\lambda_{2}+\lambda_{3}$. Since we have case (28), one of the denominators is at least $\left|n_{1}\right|\left|n_{2}+n_{3}\right|^{1 / 2}$.

Case (i): $\left|\lambda-n^{3}+c n\right|^{1 / 2} \geqq\left|n_{1}\right|\left|n_{2}+n_{3}\right|^{1 / 2}$.

Write (45) as

$$
\sum_{n_{1}+n_{2}+n_{3}=n} \int d \lambda_{1} d \lambda_{2} d \lambda_{3}\left\{d(\lambda) \cdot\left(1+\left|n_{1}\right|\right)^{s_{2}}\left|\widehat{u}\left(n_{1}, \lambda_{1}\right)\right| \frac{\left|\widehat{u}\left(n_{2}, \lambda_{2}\right)\right|\left|\widehat{u}\left(n_{3}, \lambda_{3}\right)\right|}{1+\left|n_{2}+n_{3}\right|^{1 / 2}}\right\},
$$

where $\left\{\left(1+\left|n_{1}\right|\right)^{s_{2}}\left|\widehat{u}\left(n_{1}, \lambda_{1}\right)\right|\right\}$ is controlled in $\ell_{n_{1}}^{\infty} L_{\lambda_{1}}^{1}$, taking into account the second term in (21), $\left\{\left(1+\left|n_{2}\right| s_{1}\right)\left|\widehat{u}\left(n_{2}, \lambda_{2}\right)\right|\right\}$ and $\left\{\left(1+\left|n_{3}\right|^{s_{1}}\right)\left|\widehat{u}\left(n_{3}, \lambda_{3}\right)\right|\right\}$ are in $\ell_{n}^{2} L_{\lambda}^{1}$ by $(20)$. Consequently $\left\{\frac{\widehat{u}\left(n_{2}, \lambda_{2}\right)\left|\widehat{u}\left(n_{3}, \lambda_{3}\right)\right|}{1+\left|n_{2}+n_{3}\right|^{1 / 2}}\right\}$ is in $\ell_{n_{2} n_{3}}^{1} L_{\lambda_{2} \lambda_{3}}^{1}$ provided $\left\{\frac{1}{n_{2}^{s_{1}} n_{3}^{s_{1}}\left|n_{2}-n_{3}\right|^{1 / 2}}\right\}_{n_{2}, n_{3}>0}$ is in $\ell_{n_{2} n_{3}}^{2}$, which is the case for $s_{1}>\frac{1}{4}$.

Case (ii): $\left|\lambda_{1}-n_{1}^{3}+c n_{1}\right|^{1 / 2} \geqq\left|n_{1}\right|\left|n_{2}+n_{3}\right|^{1 / 2}$.

Write (45) as

$$
\sum_{n_{1}+n_{2}+n_{3}=n} \int d \lambda_{1} d \lambda_{2} d \lambda_{3} \frac{d(\lambda)}{1+\left|\lambda-n^{3}+c n\right|^{1 / 2}}\left(1+\left|n_{1}\right|\right)^{s_{2}} c\left(n_{1}, \lambda_{1}\right) \frac{\left|\widehat{u}\left(n_{2}, \lambda_{2}\right)\right|\left|\widehat{u}\left(n_{3}, \lambda_{3}\right)\right|}{1+\left|n_{2}+n_{3}\right|^{1 / 2}} \text {. }
$$

Here again $\left\{\frac{\left|\widehat{u}\left(n_{2}, \lambda_{2}\right)\right| \widehat{u}\left(n_{3}, \lambda_{3}\right) \mid}{1+\left|n_{2}+n_{3}\right|^{1 / 2}}\right\}$ is in $\ell_{n_{2} n_{3}}^{1} L_{\lambda_{2} \lambda_{3}}^{1}$; for fixed $\lambda_{2}, \lambda_{3},\left\{d\left(\lambda_{1}+\lambda_{2}+\lambda_{3}\right)\right\}$ is in $L_{\lambda_{1}}^{2}$ and $\left(1+\left|n_{1}\right|\right)^{s} c\left(n_{1}, \lambda_{1}\right)$ in $L_{\lambda_{1}}^{2}$ and $\ell_{n_{1}}^{\infty} L_{\lambda_{1}}^{2}$ and thus the product in $\ell_{n_{1}}^{\infty} L_{\lambda_{1}}^{1}$.

Case (iii): $\left|\lambda_{2}-n_{2}^{3}+c n_{2}\right|^{1 / 2} \geqq\left|n_{1}\right|\left|n_{2}+n_{3}\right|^{1 / 2}$.

Write (45) as

$$
\begin{gathered}
\sum_{n_{1}+n_{2}+n_{3}=n} \int d \lambda_{1} d \lambda_{2} d \lambda_{3} \frac{d(\lambda)}{1+\left|\lambda-n^{3}+c n\right|^{1 / 2}} \\
\left(1+\left|n_{1}\right|\right)^{s_{2}}\left|\widehat{u}\left(n_{1}, \lambda_{1}\right)\right| \frac{c\left(n_{2}, \lambda_{2}\right)}{1+\left|n_{2}+n_{3}\right|^{1 / 2}}\left|\widehat{u}\left(n_{3}, \lambda_{3}\right)\right|,
\end{gathered}
$$


where $\left\{\left(1+\left|n_{1}\right|^{s_{2}}\right) \mid \widehat{u}\left(n_{1}, \lambda_{1} \mid\right\}\right.$ is in $\ell_{n_{1}}^{\infty} L_{\lambda_{1}}^{1},\left\{\left(1+\left|n_{2},\right|^{s_{1}}\right) c\left(n_{2}, \lambda_{2}\right)\right\}$ in $\ell_{n_{2}}^{2} L_{\lambda_{2}}^{2}$,

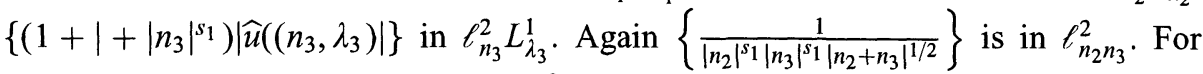
fixed $\lambda_{1}, \lambda_{3},\left\{d\left(\lambda_{1}+\lambda_{2}+\lambda_{3}\right)\right\}$ is in $L_{\lambda_{2}}^{2}$.

This completes the analysis of the contribution of (17) to the first term in (25). Clearly the preceding yields also the estimate on the second term, since the denominator $\left|\lambda-n^{3}+c n\right|$ was not used except in case (i) above where $d(\lambda)$ is replaced by $\frac{1}{1+\left|\lambda-n^{3}+c n\right|^{1 / 2}} \leqq 1$.

Next analyse the contribution of $(18)$ to $\widehat{w}(n, \lambda)$. It is clear that $(23),(24),(25)$ are bounded by $\left(\sum_{n}(1+|n|)^{2 s_{2}} \int d \lambda|\widehat{w}(n, \lambda)|^{2}\right)^{1 / 2}$, hence, from $(18)$, by

$$
|n|^{1+s_{2}}\left|\widehat{u}\left(n, \lambda_{1}\right)\right|\left|\widehat{u}\left(n, \lambda_{2}\right)\right|\left|\widehat{u}\left(-n, \lambda-\lambda_{1}-\lambda_{2}\right)\right|
$$

in $\ell_{n}^{2} L_{\lambda}^{2} L_{\lambda_{1} \lambda_{2}}^{1}$. The factors $\left\{|n|^{s_{1}}\left|\widehat{u}\left(n, \lambda_{1}\right)\right|\right\},\left\{|n|^{s_{2}}\left|\widehat{u}\left(n, \lambda_{2}\right)\right|\right\},\left\{|n|^{s_{2}}|\widehat{u}(-n, \lambda)|\right\}$ are respectively in $\ell_{n}^{2} L_{\lambda_{1}}^{1}, \ell_{n}^{\infty} L_{\lambda_{2}}^{1}, \ell_{n}^{\infty} L_{\lambda}^{2}$, by (20), (21). Hence, it suffices to ensure that

$$
s_{1}+2 s_{2}>1+s_{2}, \text { hence } s_{1}+s_{2}>1 \text {. }
$$

With $s_{1}=\frac{9}{20}, s_{2}=\frac{4}{5}$ (cf. above) this is clearly satisfied.

From the preceding, it follows that the transformation

$$
u \mapsto S(t) \phi+\int_{0}^{t} S(t-\tau) w(\tau) d \tau ; w=\left[-u^{2}+\int u^{2} d x\right] \partial_{x} u,
$$

$\phi$ with small (22)-norm, maps a ball in \|\|$\|_{s_{1}, s_{2}}$-space into itself and acts as a contraction there (proving the contractive property is a straightforward variant of the previous argument), for certain $0<s_{1}<\frac{1}{2}<s_{2}<1$. To deal with general data (without) smallness assumption, one exploits small time intervals $[0, \tau]$, to gain an extra factor $\tau^{\delta}$, for some $\delta>0$, on the second term in (51). This is possible, since in fact, as a consequense of (30), there is for $\varepsilon>0$ some $p(\varepsilon)>6$ satisfying

$$
\begin{gathered}
\left\|\sum_{N_{0}<n<N_{0}+N} e^{i n x} \int d \lambda e^{i \lambda t} \widehat{u}(n, \lambda)\right\| \|_{L_{x}^{p}(\mathbb{T}) L_{t}^{p}(\mathrm{loc})} \\
C N^{\varepsilon}\left[\sum_{n} \int d \lambda\left(1+\left|\lambda-n^{3}+c n\right|\right)|\widehat{u}(n, \lambda)|^{2}\right]^{1 / 2} .
\end{gathered}
$$

This is easily derived from (30) if $\log \left(1+\left|\lambda-n^{3}+c n\right|\right) \lesssim \log N$, breaking up in level sets $\left|\lambda-n^{3}+c n-k\right| \leqq 1$. If $\log \left|\lambda-n^{3}+c n\right| \gg \log N$, simply use a Hausdorff-Young inequality.

Details on these matters appear in [B1] in the discusision of the NLSE. This yields us a local wellposedness result for periodic modified $\mathrm{KdV}(10)$ with data $\phi$ satisfying

$$
\|\phi\|_{s_{1}, s_{2}} \equiv\|\phi\|_{H^{s_{1}(\mathbb{T})}}+\sup _{n}\left(1+|n|^{s_{2}}\right)|\widehat{\phi}(n)|<\infty
$$

for certain $0<s_{1}<\frac{1}{2}<s_{2}<1$. The time interval $[0, \tau]$ depends again on the size $\|\phi\|_{s_{1}, s_{2}}$ of the data in a polynomial way. As mentioned earlier, $\|\phi\|_{s_{1}, s_{2}}<K$ on a subset $\Omega_{s_{1}, s_{2}, K}$ of the Wiener space with complementary measure $<e^{-c K^{2}}$. 
This discussion yields all elements to carry out the scheme of proof used for the NLSE with $p>4$ in the case of modified $\mathrm{KdV}$, with the same conclusion.

Theorem 2. The Gibbs measure for $K d V$ and modified KdV on the statistical ensemble (with some $L^{2}$-cutoff if necessary) is invariant under the flow. The equations are wellposed (globally) almost everywhere on the ensemble.

\section{References}

[Bid] Bidegaray, B.: Mesures invariantes pour des équations aux dérivées partielles. Preprint Orsay

[B1] Bourgain, J.: Fourier restriction phenomena for certain lattice subsets and applications to nonlinear evolution equations. Geom. and Funct. Anal. 3, $N^{\circ} 2,107-156,209-262$ (1993)

[B2] Bourgain, J.: On the longtime behaviour of nonlinear Hamiltonian evolution equations. Preprint IHES 4/94, to appear in Geom. and Funct. Anal. (GAFA)

[B3] Bourgain, J.: Invariant measures for the $2 D$-defocusing nonlinear Schrödinger equation. Preprint IHES 4/94

[G-V] Ginibre, J., Velo, G.: Ann. Inst. H. Poincaré 28, 287-316 (1978)

[MCK-V] Mckean, H., Vaninski, K.: Statistical mechanics of nonlinear wave equations and brownian motion with restoring drift: The petit and micro-canonical ensembles. Commun. Math. Phys. 160, 615-630 (1994) and preprints 1993

[ML-S] MacLaughlin, D., Schober, C.: Chaotic and homoclinic behavior for numerical discretization of the nonlinear Schrödinger equation. Physica D 57, 447-465 (1992)

[L-R-S] Lebowitz, J., Rose, R., Speer E.: Statistical mechanics of the nonlinear Schrödinger equation. J. Stat. Phys. V 50, 657-687 (1988)

[Zh1] Zhidkov, P.: On the invariant measure for the nonlinear Schrödinger equation. Doklady Akad Nauk SSSR 317, $\mathrm{N}^{\circ}$ 3, 543 (1991)

[Zh2] Zhidkov, P.: An invariant measure for a nonlinear wave equation. Preprint 1992 